\title{
O cuidado de enfermagem à mulher com depressão pós-parto na atenção primária à saúde
}

Nursing care for women with postpartum depression in primary health care

Atención de enfermería a la mujer con depresión posparto en la atención primaria de salud

Recebido: 16/01/2022 | Revisado: 26/01/2022 | Aceito: 27/01/2022 | Publicado: 28/01/2022

Marília Girão de Oliveira Machado

ORCID: https://orcid.org/0000-0001-7163-9061

Universidade de Fortaleza, Brasil

E-mail: mariliagirao05@ hotmail.com

Caroline Dantas dos Santos

ORCID: https://orcid.org/0000-0002-6334-587X

Universidade de Fortaleza, Brasil

E-mail: krolynedantas@hotmail.com

Thaís Milene Rocha

ORCID: https://orcid.org/0000-0002-4536-3994

Universidade de Fortaleza, Brasil

E-mail: thaismilenerocha@ gmail.com

Antônia Maria Ferreira de Souza

ORCID: https://orcid.org/0000-0002-4295-9995

Universidade de Fortaleza, Brasil

E-mail: toinha_120@hotmail.com

Analayde Lima de Azevedo

ORCID: https://orcid.org/0000-0002-8782-7478

Universidade de Fortaleza, Brasil

E-mail: aanalayde@gmail.com

Márcia Maria de Oliveira Maia Freire

ORCID: https://orcid.org/0000-0003-1703-7398

Universidade de Fortaleza, Brasil

E-mail: marciamariamaia1@ hotmail.com

Luize Caroline Sampaio de Oliveira

ORCID: https://orcid.org/0000-0002-7787-2523

Universidade de Fortaleza, Brasil

E-mail: louisecarol25@yahoo.com.br

Greicy Machado Aguiar de Albuquerque ORCID: https://orcid.org/0000-0003-0787-5531 Universidade de Fortaleza, Brasi

E-mail: greicymaxado2008@hotmail.com

Suzana Aires Barbosa

ORCID: https://orcid.org/0000-0003-3895-4902 Universidade de Fortaleza, Brasil

E-mail: suzana.airesbarbosa@gmail.com

Bárbarah Nogueira Rebouças

ORCID: https://orcid.org/0000-0002-9783-3871

Universidade de Fortaleza, Brasil

E-mail: barbarahnr@hotmail.com

Aline Nabuco Morel

ORCID: https://orcid.org/0000-0003-0777-7309 Universidade de Fortaleza, Brasil

E-mail: alinenabucomorel@gmail.com

Samira Rodrigues Aguiar Palhano ORCID: https://orcid.org/0000-0001-7552-1984 Universidade de Fortaleza, Brasil

E-mail: samira.aguiar@gmail.com

Ana Carolina Teófilo Pontes

ORCID: https://orcid.org/0000-0001-8139-2760

Universidade de Fortaleza, Brasil

E-mail: carolteofilop@gmail.com

Sandraneide Pinheiro de Freitas ORCID: https://orcid.org/0000-0002-5420-4995 Universidade de Fortaleza, Brasil

E-mail: sandra_pimheiro2010@ hotmail.com 


\title{
Resumo
}

A depressão pós-parto é a condição psiquiátrica mais comum e incapacitante durante o puerpério e é por meio da atenção primária à saúde que os enfermeiros atuam na identificação dos sintomas, fornecendo um cuidado integral e humanizado. O objetivo deste estudo foi identificar, por meio de uma revisão integrativa de literatura, como se dá o cuidado de enfermagem à mulher com depressão pós-parto na atenção primária à saúde. Trata-se de uma revisão integrativa de literatura nas bases de dados: BDENF, SciELO, MEDLINE e Web of Science, por meio dos descritores: Atenção Primária à Saúde; Depressão Pós-parto e Cuidado de Enfermagem. Os critérios de inclusão foram: artigos completos, dissertações ou teses, publicados nos últimos cinco anos (2016 a 2021), nos idiomas português, inglês ou espanhol. Foram excluídas monografias, cartas ao editor, revisões integrativas e pesquisas que não estavam disponíveis na íntegra eletronicamente. Foram identificados o total de 11 artigos, agrupados em duas categorias temáticas: estratégias de cuidado de enfermagem à mulher com depressão pós-parto e desafios do cuidado de enfermagem à mulher com depressão pós-parto na atenção primária à saúde. Foi enfatizada a importância do cuidado de enfermagem por meio de ações de consulta de enfermagem, visita domiciliar, apoio matricial, entre outras. Evidenciou-se escassez no conhecimento de enfermeiros, predominância do modelo biomédico e cuidado fragmentado, resultando em dificuldade no diagnóstico precoce, sinalizando a necessidade de educação permanente de enfermeiros, visando fornecer um atendimento integral e de qualidade.

Palavras-chave: Cuidado de enfermagem; Atenção primária à saúde; Depressão pós-parto; Saúde mental.

\begin{abstract}
Postpartum depression is the most common and disabling psychiatric condition during the puerperium and it is through primary health care that nurses work to identify symptoms, providing comprehensive and humanized care. The objective of this study was to identify, through an integrative literature review, how nursing care is given to women with postpartum depression in primary health care. This is an integrative literature review in the following databases: BDENF, SciELO, MEDLINE and Web of Science, using the descriptors: Primary Health Care; Postpartum Depression and Nursing Care. Inclusion criteria were: complete articles, dissertations or theses, published in the last five years (2016 to 2021, in Portuguese, English or Spanish. Monographs, letters to the editor, integrative reviews and research that were not available in full electronically were excluded A total of 11 articles were identified, grouped into two thematic categories: nursing care strategies for women with postpartum depression and challenges of nursing care for women with postpartum depression in primary health care. of nursing care through nursing consultation actions, home visits, matrix support, among others. There was a lack of knowledge of nurses, predominance of the biomedical model and fragmented care, resulting in difficulty in early diagnosis, signaling the need for permanent education of nurses, aiming to provide comprehensive and quality care.
\end{abstract}

Keywords: Nursing care; Primary health care; Postpartum depression; Mental health.

\section{Resumen}

La depresión posparto es la condición psiquiátrica más común e incapacitante durante el puerperio y es a través de la atención primaria de salud que las enfermeras trabajan para identificar los síntomas, brindando una atención integral y humanizada. El objetivo de este estudio fue identificar, a través de una revisión integradora de la literatura, cómo se brinda el cuidado de enfermería a las mujeres con depresión posparto en la atención primaria de salud. Se trata de una revisión integrativa de la literatura en las siguientes bases de datos: BDENF, SciELO, MEDLINE y Web of Science, utilizando los descriptores: Atención Primaria en Salud; Depresión posparto y cuidados de enfermería. Los criterios de inclusión fueron: artículos completos, disertaciones o tesis, publicados en los últimos cinco años (2016 a 2021), en portugués, inglés o español. Se excluyeron monografías, cartas al editor, revisiones integradoras e investigaciones que no estaban disponibles en su totalidad electrónicamente. Fueron identificados un total de 11 artículos, agrupados en dos categorías temáticas: estrategias de atención de enfermería a la mujer con depresión posparto y desafíos de la atención de enfermería a la mujer con depresión posparto en la atención primaria de salud del cuidado de enfermería a través de acciones de consulta de enfermería, visita domiciliaria, matriz de apoyo, entre otros, hubo desconocimiento de los enfermeros, predominio del modelo biomédico y atención fragmentada, resultando en dificultad en el diagnóstico precoz, señalando la necesidad de educación permanente de los enfermeros, con el objetivo de brindar un cuidado integral y de calidad.

Palabras clave: Cuidado de enfermería; Primeros auxílios; Depresión posparto; Salud mental.

\section{Introdução}

Segundo a Organização Mundial de Saúde (OMS, 2011), aproximadamente 73 milhões de mulheres estão suscetíveis 
a eventos depressivos a cada ano, de maneira que, $13 \%$ dessas mulheres desencadeiam depressão após o parto. Dessa forma, a Depressão Pós-Parto (DPP) é a condição psiquiátrica mais comum e incapacitante durante o puerpério, constituindo um problema de saúde pública, com prevalência entre 10\% e 20\% em todo o mundo (Souza et al., 2018).

A prevalência de DPP no Brasil é cerca de $26 \%$ mais elevada que a média estimada pela OMS para países de baixa renda. Aproximadamente $25 \%$ das puérperas apresentam sintomas de depressão no período de seis a 18 meses pós-parto, todavia, a prevalência de DPP pode divergir conforme o instrumento de avaliação adotado, o período de coleta de dados, o tipo de amostra e aculturação (Teixeira et al., 2021; Theme Filha et al., 2016).

Trata-se de uma doença de etiologia multifatorial, cursando com alterações emocionais, cognitivas, comportamentais e físicas, podendo se agravar para uma psicose. Os sintomas mais comuns da DPP são: tristeza, culpa, alterações do sono, cansaço, desamparo, anedonia, baixa autoestima, perda ou ganho significativo de peso, retardo ou agitação psicomotora, dificuldade de concentração, ideação e pensamento suicida. Destacam-se também, alterações no humor, irritabilidade, ansiedade, sentimento de inutilidade e preocupações obsessivas, geralmente associadas aos cuidados com o bebê (Moll et al., 2019; Santos et al., 2020; Stewart \& Vigod, 2019).

Considerando a importância da Atenção Primária à Saúde (APS) como porta de entrada na realização de ações de promoção, prevenção e cuidado no ciclo gravídico-puerperal, é por meio da assistência de enfermagem que os enfermeiros atuam na identificação dos sintomas de DPP, fornecendo um cuidado integral e humanizado (Araújo et al., 2020; Silva et al., 2021; Gonçalves et al., 2020).

No entanto, muitos enfermeiros da APS apresentam barreiras na identificação e manejo de casos de DPP, além da escassez de capacitação profissional. Tais barreiras podem gerar fragilidades na atenção dispensada às puérperas em sofrimento psíquico. Deste modo, faz-se necessário que haja uma reorganização da APS, no intuito de que estes serviços contemplem uma assistência integral e de qualidade (Araújo et al., 2020; Santos et al., 2020; Gonçalves et al., 2020).

Diante do exposto, os achados desta revisão objetivaram identificar como se dá o cuidado de enfermagem frente à mulher com DPP na APS. O presente estudo justifica-se por propor melhorias nos serviços APS e contribuir com a expansão do conhecimento na comunidade acadêmica e científica, possibilitando a disseminação do conhecimento e da compreensão do sofrimento psíquico no puerpério. Assim, será possível minimizar danos relacionados ao binômio mãe-filho e promover uma assistência de enfermagem mais humanizada.

Desse modo, o objetivo deste estudo foi identificar como se dá o cuidado de enfermagem à mulher com DPP na APS.

\section{Metodologia}

Para o desenvolvimento deste estudo, foi realizada uma Revisão Integrativa da Literatura. Segundo Mendes, Silveira e Galvão (2008), trata-se de um método que consiste na síntese de múltiplos estudos, contribuindo para a tomada de decisão profissional na prática clínica. Para Polit e Beck (2019), esse método consiste em reunir e aprofundar evidências sobre determinado assunto de forma sistemática, incluindo as seguintes fases: formulação de uma questão, definição de uma estratégia de pesquisa, busca e armazenamento de fontes relevantes, resumo, codificação, análise e preparo das informações.

Para nortear a busca das publicações científicas, a pergunta desta pesquisa foi formulada a partir da estratégia PICO (Milner \& Cosme, 2017): quais os conhecimentos produzidos sobre o cuidado de enfermagem à mulher com DPP na APS? Onde $\mathrm{P}=$ mulher com DPP; $\mathrm{I}=$ cuidado de enfermagem, $\mathrm{C}=$ APS e $\mathrm{O}=$ conhecimentos produzidos.

Foram utilizados os Descritores em Ciências da Saúde (DeCS) e o Medical Subject Headings (MESH), os quais foram todos agrupados utilizando-se o operador booleano AND: Atenção Primária à Saúde (Primary Health Care) AND Depressão Pós-parto (Depression Postpartum) AND Cuidado de Enfermagem (Nursing Care), por meio de uma busca on-line pelas seguintes bases de dados: Bases de dados de Enfermagem (BDENF), Scientific Electronic Library Online (SciELO), Medical 


\section{Literature Analysis and Retrieval System Online (MEDLINE) e Web of Science.}

Os critérios de inclusão utilizados foram: artigos completos, dissertações ou teses, publicados nos últimos cinco anos (2016 a 2021), por utilizar referências mais atuais, nos idiomas português, inglês ou espanhol que atendiam à temática estabelecida. Foram excluídas monografias, cartas ao editor, revisão integrativa e pesquisas que não estavam disponíveis na íntegra eletronicamente. O processo de seleção das pesquisas ocorreu inicialmente através de leitura minuciosa de títulos e resumos e foram selecionadas as que atenderam aos critérios de inclusão já mencionados. A busca de artigos ocorreu durante o mês de junho de 2021.

Ressalta-se que este é um recorte de uma dissertação de mestrado, onde foi realizada uma revisão de literatura, que compreendeu uma das fases do estudo sobre o desenvolvimento e validação de uma tecnologia assistencial para a consulta de enfermagem à mulher com manifestações de DPP na APS (Luz, 2021).

Por se tratar de uma revisão integrativa de literatura, o estudo não foi submetido ao Comitê de Ética em Pesquisa (CEP).

\section{Resultados}

No total 373 artigos foram encontrados nas bases de dados eletrônicas. Com base na utilização dos critérios de busca definidos, foram incluídos 11 artigos, sendo cinco na base de dados BDENF, um na SCIELO, dois na MEDLINE e quatro na WEB OF SCIENCE, publicados entre 2016 e 2021. Um estudo duplicou-se nas bases de dados SCIELO e MEDLINE.

Para descrição das buscas e seleções do estudo, utilizou-se o Preferred Reporting Items for Systematic Review and Meta-Analyses (PRISMA), conforme a Figura 1.

Figura 1 - Fluxograma de seleção de artigos, Fortaleza, Ceará, Brasil, 2022.

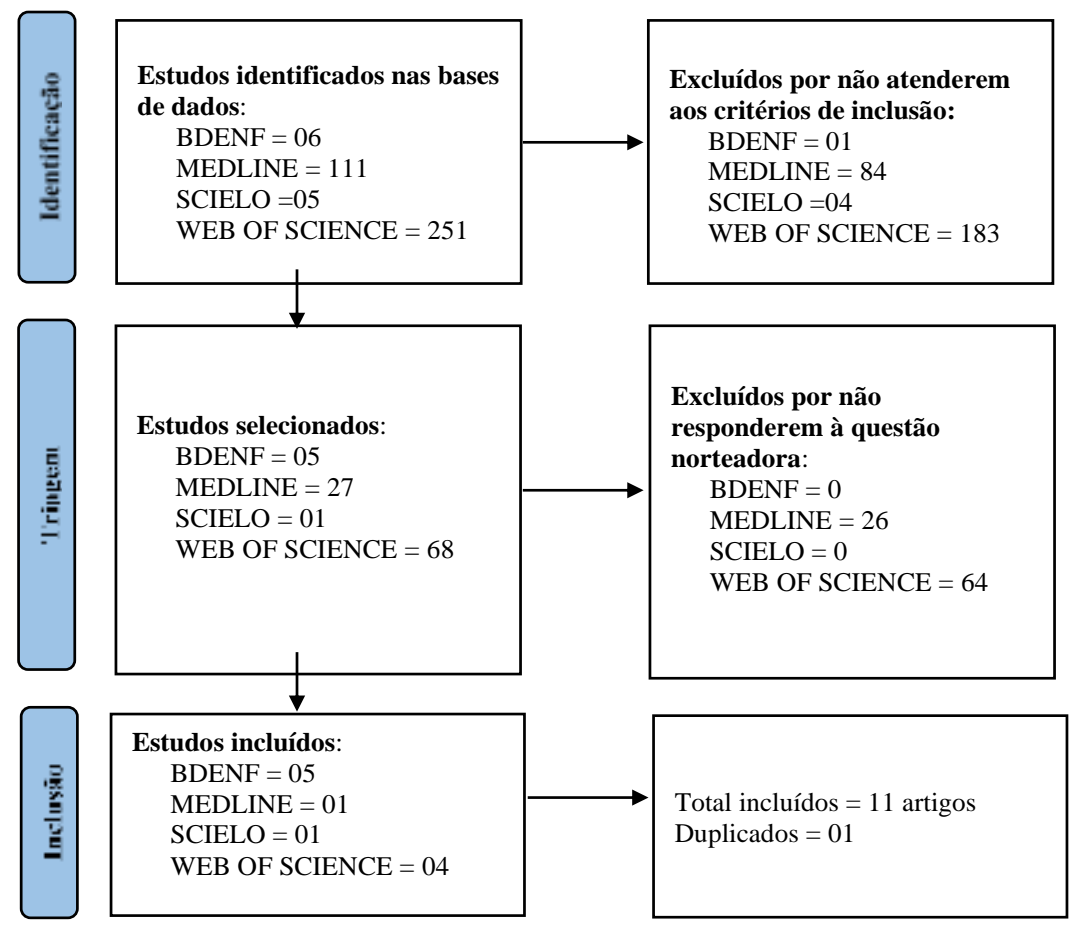

Fonte: Elaborado pelos autores (2022).

Com base na busca na literatura, seis artigos são de origem nacional, todos publicados em revistas de enfermagem por meio de estudos transversais, ensaios clínicos randomizados e estudos de coorte, com origem nas regiões nordeste, sul e 
sudeste do país. Os estudos internacionais foram publicados em diferentes países, com destaque para ensaios clínicos randomizados. As produções permitiram descrever sobre diferentes formas do cuidado de enfermagem às mulheres com DPP nos serviços de APS, conforme Quadro 1.

Quadro 1 - Sinopse dos artigos selecionados. Fortaleza, Ceará - 2022. n= 11.

\begin{tabular}{|c|c|c|c|c|}
\hline Periódico/Base de dados & Autor(es)/ano & Objetivo & Tipo de estudo & Local do estudo \\
\hline $\begin{array}{c}\text { Nursing (São Paulo / } \\
\text { BDENF }\end{array}$ & $\begin{array}{l}\text { Santos et al. } \\
\quad(2020)\end{array}$ & $\begin{array}{l}\text { Analisar as percepções de enfermeiros } \\
\text { sobre diagnóstico e acompanhamento } \\
\text { da DPP em Divinópolis-MG }\end{array}$ & Descritivo, qualitativo & Divinópolis-MG \\
\hline $\begin{array}{l}\text { Revista enfermagem } \\
\text { UFPE on line / BDENF }\end{array}$ & $\begin{array}{l}\text { Moll et al. } \\
\text { (2019) }\end{array}$ & $\begin{array}{l}\text { Rastrear a DPP entre mulheres jovens } \\
\text { que estão na segunda semana e no sexto } \\
\text { mês após o parto }\end{array}$ & $\begin{array}{l}\text { Descritivo, } \\
\text { exploratório e } \\
\text { transversal, } \\
\text { quantitativo }\end{array}$ & $\begin{array}{l}\text { Interior de Minas } \\
\text { Gerais }\end{array}$ \\
\hline $\begin{array}{l}\text { Revista enfermagem } \\
\text { UFPE on line / BDENF }\end{array}$ & $\begin{array}{l}\text { Souza et al. } \\
\quad(2018)\end{array}$ & $\begin{array}{l}\text { Analisar o conhecimento dos } \\
\text { enfermeiros das unidades de saúde da } \\
\text { família sobre a depressão puerperal. }\end{array}$ & Descritivo, qualitativo & Guanambi/BA \\
\hline $\begin{array}{l}\text { Journal of Nursing and } \\
\text { Health / BDENF }\end{array}$ & $\begin{array}{l}\text { Oliveira et al. } \\
\quad \text { (2016) }\end{array}$ & $\begin{array}{l}\text { Investigar o conhecimento de } \\
\text { profissionais da Estratégia Saúde da } \\
\text { Família quanto ao tratamento da DPP }\end{array}$ & Descritivo, qualitativo & Caicó/RN \\
\hline $\begin{array}{l}\text { Journal of nursing and } \\
\text { health / BDENF }\end{array}$ & $\begin{array}{l}\text { Teixeira et al. } \\
\quad \text { (2021) }\end{array}$ & $\begin{array}{l}\text { Detectar a prevalência de DPP e fatores } \\
\text { sociodemográficos em } \\
\text { puérperas atendidas em uma unidade } \\
\text { por equipes de Saúde da Família. }\end{array}$ & $\begin{array}{l}\text { Observacional } \\
\text { descritivo, } \\
\text { quantitativa }\end{array}$ & Teresina/PI \\
\hline $\begin{array}{l}\text { Cadernos de Saúde Pública } \\
\text { / SCIELO / MEDLINE }\end{array}$ & $\begin{array}{l}\text { Baratieri, } \\
\text { Natal e Hartz } \\
\quad(2020)\end{array}$ & $\begin{array}{l}\text { Desenvolver e sistematizar um modelo } \\
\text { avaliativo da assistência às mulheres no } \\
\text { pós-parto na APS, verificando sua } \\
\text { avaliabilidade }\end{array}$ & $\begin{array}{l}\text { Estudo de } \\
\text { Avaliabilidade, } \\
\text { qualitativo }\end{array}$ & $\begin{array}{l}\text { Paraná, Santa } \\
\text { Catarina e Rio } \\
\text { Grande do Sul }\end{array}$ \\
\hline $\begin{array}{l}\text { The British Journal of } \\
\text { Psychiatry / MEDLINE }\end{array}$ & $\begin{array}{l}\text { Dennis et al. } \\
\quad(2020)\end{array}$ & $\begin{array}{l}\text { Examinar a eficácia da psicoterapia } \\
\text { interpessoal por telefone administrada } \\
\text { por enfermeiros para a DPP }\end{array}$ & $\begin{array}{l}\text { Ensaio clínico } \\
\text { randomizado }\end{array}$ & Canadá \\
\hline $\begin{array}{l}\text { Revista Latino-americana } \\
\text { de Enfermagem/ } \\
\text { WEB OF SCIENCE }\end{array}$ & $\begin{array}{l}\text { Martínez; } \\
\text { Vöhringer e } \\
\text { Rojas (2016) }\end{array}$ & $\begin{array}{l}\text { Desenvolver um modelo preditivo para } \\
\text { avaliar os fatores que modificam o } \\
\text { acesso a tratamento para a DPP }\end{array}$ & $\begin{array}{l}\text { Estudo de coorte } \\
\text { prospectivo, } \\
\text { quantitativo }\end{array}$ & $\begin{array}{l}\text { Região } \\
\text { metropolitana do } \\
\text { Chile }\end{array}$ \\
\hline $\begin{array}{c}\text { Journal of perinatal } \\
\text { education/ } \\
\text { WEB OF SCIENCE }\end{array}$ & $\begin{array}{l}\text { Bendavid; } \\
\text { Hunker e } \\
\text { Spadaro (2016) }\end{array}$ & $\begin{array}{l}\text { Implementar a triagem por telefone em } \\
\text { uma prática de atenção primária urbana } \\
\text { que atende uma população vulnerável }\end{array}$ & $\begin{array}{l}\text { Descritivo, } \\
\text { quantitativo }\end{array}$ & Estados Unidos \\
\hline $\begin{array}{l}\text { Nursing and Midwifery } \\
\text { Studies/ } \\
\text { WEB OF SCIENCE }\end{array}$ & $\begin{array}{l}\text { Nasiri et al. } \\
\quad(2018)\end{array}$ & $\begin{array}{l}\text { Comparar o efeito da terapia de } \\
\text { resolução de problemas e do do } \\
\text { relaxamento na gravidade dos sintomas } \\
\text { de DPP }\end{array}$ & $\begin{array}{l}\text { Ensaio clínico } \\
\text { randomizado, } \\
\text { quantitativo }\end{array}$ & Mashhad, Irã \\
\hline $\begin{array}{c}\text { PLoS One/ } \\
\text { WEB OF SCIENCE }\end{array}$ & $\begin{array}{l}\text { Tandon et al. } \\
\quad(2020)\end{array}$ & $\begin{array}{l}\text { Examinar os resultados relacionados à } \\
\text { triagem de DPP, recebimento de } \\
\text { serviços baseados em evidências e } \\
\text { reduções nos sintomas depressivos } \\
\text { entre clientes de programas de visitas } \\
\text { domiciliares }\end{array}$ & $\begin{array}{l}\text { Ensaio clínico } \\
\text { randomizado, } \\
\text { quantitativo }\end{array}$ & Estados Unidos \\
\hline
\end{tabular}

Fonte: Bases de dados BDENF, SCIELO, MEDLINE e WEB OF SCIENCE. Fortaleza, Ceará, Brasil (2022).

Deste modo, buscou-se agrupar as discussões semelhantes em duas categorias temáticas: estratégias de cuidado de enfermagem à mulher com DPP na APS e desafios do cuidado de enfermagem à mulher com DPP na APS.

\section{Discussões}

\subsection{Estratégias de cuidado de enfermagem à mulher com DPP na APS}

A enfermagem possui papel fundamental na prevenção, detecção e tratamento da DPP, reconhecendo os fatores de risco e atuando no planejamento de atividades de promoção à saúde dentro de uma perspectiva psicossocial (Moll et al., 2019; Santos et al., 2020). Santos et al. (2020), ressaltam que o cuidado de enfermagem deve ser planejado em uma perspectiva multidisciplinar que envolva ações de referência e contrarreferência. 
No Nordeste do Brasil, autores detectaram alta prevalência de DPP em puérperas atendidas na APS (Teixeira et al., 2021). Estudo semelhante realizado no interior de Minas Gerais, identificou prevalência de 19,7\% de DPP em 66 mulheres de 18 a 26 anos, entre a segunda semana e o sexto mês pós-parto (Moll et al., 2019). Considerando o exposto, a APS consiste em um importante componente da Rede de Atenção à Saúde (RAS) com potencialidades para fornecer um cuidado efetivo à mulher com DPP. Tais resultados apontam para a necessidade da detecção precoce de DPP na APS e a valorização dos aspectos individuais e sociodemográficos das puérperas, buscando estabelecer um cuidado de enfermagem efetivo e em tempo oportuno.

Frente a isso, a consulta de enfermagem no puerpério pode ocorrer durante a visita domiciliar ou no serviço de saúde, no período de 42 dias após o parto, possibilitando o acolhimento e encaminhamentos necessários aos demais níveis de atenção à saúde (Santos et al., 2020). Para Souza et al. (2018) é na consulta puerperal que o enfermeiro avalia os fatores biopsicossociais da puérpera e o vínculo mãe-filho. As intercorrências devem ser verificadas nesse momento e prestadas as devidas orientações com registros realizados no prontuário.

Destarte, o cuidado de enfermagem é realizado por meio de uma abordagem integral e terapêutica capaz de identificar as transformações ocorridas no processo saúde-doença da mulher em sofrimento psíquico. Assim, foram elaboradas estratégias para o cuidado, incluindo a utilização de instrumentos próprios de enfermagem como a Sistematização da Assistência de Enfermagem (SAE), tornando possível a operacionalização do processo de enfermagem (PE) (Souza et al., 2018; Santos et al., 2020).

No tocante à importância da visita domiciliar realizada pelo enfermeiro, Tandon et al. (2020) afirmaram que se trata de um momento oportuno para realização de triagem, prevenção e tratamento da DPP. Assim, os programas de visita domiciliar podem desempenhar um papel importante no fechamento de lacunas na identificação da depressão materna, com o objetivo de promover uma série de resultados favoráveis à saúde materno-infantil.

A visita domiciliar, conforme citada por Santos et al. (2020), deve ocorrer no período de 7 a 10 dias de pós-parto, onde são avaliadas as condições fisiológicas, psicológicas e sociais da puérpera, as condições do domicílio, o contexto socioeconômico, a relação entre os familiares, além do vínculo mãe-filho. Trata-se de um importante momento para realizar orientações e sanar dúvidas comuns no puerpério.

Para Dennis et al. (2020) as mulheres que sofrem de depressão pós-parto precisam de tratamentos oportunos, eficazes e acessíveis. Diante desse contexto, é primordial que os enfermeiros da APS estejam atentos e capacitados para manejar a DPP e possam produzir saberes específicos na área. Nesse sentindo, a disposição de estratégias e ferramentas teórico-práticas para lidar com a DPP pode proporcionar o apoio necessário para as puérperas (Martínez, Vöhringer \& Rojas, 2016).

Dessa forma, estudo realizado na região Sul do país, utilizou revisão da literatura, análise documental e entrevista com stakeholders (interessados na avaliação) e os resultados permitiram formular um modelo orientado para a assistência integral à saúde das mulheres no pós-parto. A partir disso, foi elaborada a teoria do programa com objetivos, metas, atividades, produtos, resultados e impacto no cuidado pós-parto. Assim, esse modelo contribuiu para implantar e aprimorar a assistência pós-parto na APS (Baratieri et al., 2020).

Outro instrumento de cuidado de enfermagem à mulher com DPP é o apoio matricial, que consiste em um suporte técnico-pedagógico e especializado às equipes de saúde, com integração dialógica entre distintas especialidades e profissões. Essa proposta de cuidado visa implementar a clínica ampliada, fornecendo uma assistência colaborativa entre a saúde mental e APS, a fim de ampliar seu campo de atuação e qualificar suas ações (Oliveira et al., 2016).

No que tange ao rastreio dos sintomas depressivos no puerpério, Moll et al. (2019) e Martínez et al. (2016) ressaltaram a importância da Escala de Depressão Pós-natal de Edimburgo (EPDS - Edinburgh Postnatal Depression) como um instrumento bastante utilizado por enfermeiros. Tal instrumento possui 10 itens que variam conforme a presença e 
intensidade dos sintomas. A somatória dos escores variam de 0 a 30, sendo considerados de sintomatologia depressiva o valor igual ou superior a 10 (Moll et al., 2019; Martínez et al., 2016).

No contexto internacional, Bendavid et al. (2016) utilizaram um protocolo de triagem para DPP na APS por telefone, visando identificar os sintomas de DPP entre duas e três semanas após o parto e iniciar o tratamento precoce. Os sintomas depressivos foram identificados em um número clinicamente significativo de mulheres, sendo estas encaminhadas para atendimento imediato e serviços de apoio em saúde mental.

Desse modo, um ensaio clínico randomizado realizado por enfermeiros em centros de saúde do Irã, comparou o efeito da terapia de resolução de problemas e relaxamento na gravidade dos sintomas depressivos pós-parto. Os resultados evidenciaram que ambas as intervenções foram eficazes na redução dos sintomas depressivos em mulheres com DPP e não apresentaram efeitos adversos, podendo ser utilizadas na rotina de atendimento como métodos fáceis, baratos e aplicáveis (Nasiri et al., 2018).

Assim, os achados permitiram identificar as principais estratégias relacionadas ao cuidado de enfermagem a mulher com DPP na APS, contribuindo com a busca de melhores condições de saúde e corroborando com o modelo psicossocial.

\subsection{Desafios do cuidado de enfermagem à mulher com DPP na APS}

Os enfermeiros possuem participação ativa no processo de cuidado, devendo romper com o modelo biomédico e a fragmentação do cuidado. No entanto, estudo revelou que a visita puerperal realizada pelo enfermeiro, é tecnicista e centralizada no cuidado ao recém-nascido e as alterações físicas e reprodutivas da mulher, sem levar em consideração os aspectos psíquicos e suas dimensões e necessidades de cuidado (Santos et al., 2020).

De forma semelhante, estudo realizado por Souza et al. (2018) analisaram o conhecimento dos enfermeiros da APS sobre a DPP e identificaram que o cuidado de enfermagem é centralizado no bebê, com ênfase nas ações de orientação e aleitamento materno e pouco espaço para ações voltadas às condições biopsicossociais de mulheres no pós-parto. Tais ações reforçam a necessidade de educação permanente e continuada para os profissionais da ESF, além da necessidade de investimentos em ações de saúde mental na APS (Oliveira et al., 2016).

Estudo realizado em um município de Minais Gerais, analisou que os enfermeiros não possuíam suporte literário prédefinido no atendimento de casos de DPP, sendo o psicólogo ou psiquiatra os principais responsáveis pela condução dos casos. Dentre o cuidado realizado pela enfermagem, espera-se que os enfermeiros possuam conhecimentos sobre DPP e realizem a busca ativa para prevenção e acompanhamento eficaz na APS (Santos et al., 2020).

$\mathrm{Na}$ mesma direção, Oliveira et al. (2016) investigaram o conhecimento de profissionais da ESF quanto ao tratamento da DPP e constataram que os atendimentos e tratamentos são fragmentados e pouco resolutivos, sendo uma doença limitada pelo saber médico. Dentre os desafios encontrados no cuidado de enfermagem à mulher com DPP na APS, a necessidade de educação permanente de enfermeiros da ESF, por meio de capacitações, foi descrita por muitos estudos (Souza et al., 2018; Santos et al., 2020; Oliveira et al., 2016).

A DPP pode ser difícil de ser diagnosticada pela equipe de enfermagem em um primeiro momento, devido à semelhança com sintomas de ajustamento emocional no período pós-parto. Na Bahia, foi evidenciado que os enfermeiros não sabiam a diferença de DPP e o baby blues, caracterizado por alterações de humor de intensidade leve a moderada, presente em $40 \%$ a $80 \%$ de puérperas (Souza et al., 2018). Esse achado é semelhante a estudo de Moll et al. (2019), onde enfermeiros apresentavam dificuldades na identificação da DPP, sendo os sintomas geralmente confundidos como oriundos de um período de ajustamento emocional (Moll et al., 2019).

Dennis et al. (2020) e Martinez et al. (2016) evidenciaram limitações no acesso às tecnologias para DPP, incluindo número insuficiente de especialistas em saúde mental, estigma, disponibilidade de tempo e barreiras geográficas, 
especialmente em áreas rurais carentes, com menos de 20\% de mulheres tratadas. Segundo Tandon et al. (2020), mães com DPP usam com menor frequência os serviços de saúde preventiva e mais frequentemente os serviços ambulatoriais e de internação.

Segundo Baratieri et al. (2020), existem algumas lacunas em relação às principais políticas para assistência no pósparto às mulheres, visto que, documentos nacionais não abordam a assistência pós-parto de forma integral, a exemplo da Rede Cegonha, amplamente utilizada na atenção obstétrica e neonatal, com ênfase ao materno-infantilismo. De forma similar, o Protocolo de Atenção Básica para a Saúde da Mulher descreve as atividades a serem realizadas no pós-parto de forma genérica, sem detalhes e evidências científicas das recomendações que as embasaram. Estes desafios implicam o desenvolvimento de reflexões sobre o campo da saúde mental no pós-parto.

Logo, as ações de promoção, proteção da saúde, prevenção de agravos, diagnóstico e tratamento resultantes da DPP, devem ser rotineiras, visando a redução de danos ao binômio mãe-filho. Para tanto, faz-se necessária a formulação de políticas públicas que atendam às necessidades dessa população, oportunizando uma assistência qualificada e humanizada (Souza et al., 2018; Santos et al., 2020; Nasiri et al., 2018; Moll et al., 2019; Martínez et al. 2016).

\section{Considerações Finais}

A partir da revisão de literatura, foi identificado como se ocorria o cuidado de enfermagem à mulher com DPP na APS, por meio de ações de consulta de enfermagem, visita domiciliar, apoio matricial, entre outras. Dentre os principais desafios da enfermagem na assistência à mulher com DPP, evidenciou-se escassez no conhecimento de enfermeiros, predominância do modelo biomédico e cuidado fragmentado, resultando em dificuldade no diagnóstico precoce de DPP na APS.

É necessário que atividades de educação permanente de enfermeiros sejam realizadas para o cuidado à mulher com DPP, visando fornecer um atendimento integral e de qualidade.

Salienta-se a limitação deste estudo pelo fato de que esta revisão é um recorte de uma dissertação de mestrado, onde a busca de artigos se deu no ano de 2021, o que pode limitar a generalização dos achados, sobretudo, estudos mais recentes sobre a temática abordada. Recomenda-se a realização de estudos futuros sobre a DPP e suas repercussões na saúde mental materna no contexto nacional e internacional, repensando práticas e políticas de cuidado.

\section{Referências}

Araújo, A. B. de., Nunes, Ádrya C. M., Pessoa, A. V. S., Gomes, B. C., Silva, E. R., Sousa, L. de M., Sousa, N. R. P. de., \& Soares, F. A. de F. (2020). Nursing care for women with anxiety and depression during pregnancy: an integrative review. Research, Society and Development, 9(10), e4349106961.

Baratieri, T., Natal, S., Hartz, Z. M. A. (2020). Postpartum care for women in primary care: building an assessment model. Cad. Saúde Pública 2020; 36(7):e00087319.

Bendavid, D. N., Hunker, D. F., \& Spadaro, K. C. (2016). Uncovering the Golden Veil: applying the evidence for telephone screening to detect early postpartum depression. The J Perinat Educ, 25(1)37-45.

Dennis, C., Grigoriadis, S., Zupancic, J., Kiss, A., \& Ravitz, P. (2020). Telephone-based nurse-delivered interpersonal psychotherapy for postpartum depression: Nationwide randomised controlled trial. The British Journal of Psychiatry, 216(4), 189-196.

Gonçalves, C. L da S., Oliveira, A. M. N. de, Jantara, R. D, Gomes, GC, Silva, MRS da, \& Afonso, M. do S. (2020). Conhecimento dos profissionais da estratégia saúde da família sobre a depressão pós-parto. Research, Society and Development, 9 (7), e337973842.

Luz, M. G. O. M. (2021). Tecnologia assistencial para consulta de enfermagem à mulher com manifestações de depressão pós-parto. Dissertação de mestrado, Universidade de Fortaleza, Ceará, CE, Brasil.

Martínez, P., Vöhringer, P. A., \& Rojas, G. (2016). Barriers to access to treatment for mothers with postpartum depression in primary health care centers: a predictive model. Rev. Latino-Am. Enfermagem, 24, e2675.

Mendes, K. D. S., Silveira, R. C. C. P., \& Galvão, C. M. (2008). Revisão integrativa: método de pesquisa para a incorporação de evidências na saúde e na Enfermagem. Texto Contexto - Enferm, 17(54)4, 758-64, 2008. 
Research, Society and Development, v. 11, n. 2, e37911225811, 2022

(CC BY 4.0) | ISSN 2525-3409 | DOI: http://dx.doi.org/10.33448/rsd-v11i2.25811

Milner, K. A., \& Cosme S. (2017). The PICO Game: an innovative strategy for teaching step in evidence-based practice. Worldviews Evid Based Nurs. Aug 11. [Epub ahead of print].

Moll, M. F., Matos, A., Rodrigues, T. A., Martins, T. S., Pires, F. C. \& Pires, N. A. S. (2019). Tracking Postpartum depression in Young wonen. Journal of Nursing, UFPE online, 13(5), 1338-1344.

Nasiri, S., Kordi, M., Gharavi, M. M., \& Lotfabadi, M. K (2018). Effect of problem-solving therapy and relaxation on the severity of postpartum depressive symptoms: A randomized controlled trial. Nurs Midwifery Study, 7(1)6-11, 2018.

Oliveira, A. M., Alves, T. R. M., Azevedo, A. O., Cavalcante, R. D., A., \& Azevedo, D. M. (2016). Conhecimento de profissionais da Estratégia Saúde da Família sobre depressão pós-parto. J Nurs Health, 1(1),17-26.

Organização Mundial da Saúde (2011). Mulheres e saúde: evidências de hoje, agenda de amanhã. http://www.who.int/ageing/mulheres_saude.pdf.

Polit, D.F.; Beck, C.T. (2019). Fundamentos de pesquisa em Enfermagem: avaliação de evidências para a prática da Enfermagem. Artmed.

Santos, F. K., Silva, S. C., Ariana S, M., Santos, Lago, K., Nunes A, S., \& Consolação, S. R. (2020). Percepção de enfermeiros sobre diagnóstico e acompanhamento de mulheres com depressão pós-parto. Nursing (São Paulo), 23(271), 4999-5012.

Silva, C. M., Câmara, M. A., Zanon, B. P., Donaduzzi, D. S. S., \& Anversa, E. T. R. (2021). Fatores, conhecimento, identificação de sinais e sintomas de depressão pós-parto pelos enfermeiros na atenção primária à saúde: revisão integrativa. Brazilian Journal Of Health Review, 4(2) 4005-4027.

Souza, K., Santos, A., Boa Sorte, E., Peixoto, L., \& Carvalho, B. (2018). Conhecimento de enfermeiros da atenção básica acerca da depressão puerperal. Revista de Enfermagem UFPE on line, 12(11), 2933-2943.

Stewart, D. E., \& Vigod, S. N. (2019). Postpartum Depression: Pathophysiology, Treatment, and Emerging Therapeutics. Annual review of medicine, 70, 183196.

Tandon, D., Mackrain, M., Beeber, L., Topping-Tailby, N., Raska, M., \& Arbour, M. (2020). Addressing maternal depression in home visiting: Findings from the home visiting collaborative improvement and innovation network. PloS one, 15(4), e0230211.

Teixeira, M. G., Teixeira, M. G., Carvalho, C. M. S., Magalhães, J. M., Veras, J. M. M. F., Amorim, F. C. M., Jacobina, P. K. F (2021). Detecção precoce da depressão pós-parto na atenção básica. J. Nurs. Health, 11(2).

Theme Filha, M. M., Ayers, S., da Gama, S. G., \& Leal, M. (2016). Factors associated with postpartum depressive symptomatology in Brazil: The Birth in Brazil National Research Study, 2011/2012. Journal of affective disorders, 194, 159-167. 